Carlos Cesar Laufer

\title{
Contract Oriented Web Services Model (COWS) - Um Modelo Baseado em Contratos para Suporte a Processos de Negócios na Web
}

Tese de Doutorado

Tese apresentada como requisito parcial para obtenção do título de Doutor pelo Programa de PósGraduação em Informática da PUC-Rio.

Orientador: Daniel Schwabe 
Carlos Cesar Laufer

\title{
Contract Oriented Web Services Model (COWS) - Um Modelo Baseado em Contratos para Suporte a Processos de Negócios na Web
}

Tese apresentada como requisito parcial para obtenção do título de Doutor pelo Programa de Pós-Graduação em Informática da PUC-Rio. Aprovada pela Comissão Examinadora abaixo assinada.

\author{
Daniel Schwabe \\ Orientador \\ Departamento de Informática - PUC-Rio \\ Marco Antonio Casanova \\ Departamento de Informática - PUC-Rio \\ Renato Fontoura de Gusmão Cerqueira \\ Departamento de Informática - PUC-Rio \\ Fernanda Lima \\ Centro de Ciência e Tecnologia - UCB \\ Ricardo Choren Noya \\ Seção de Engenharia de Computação - IME
}

José Eugenio Leal

Coordenador Setorial do Centro Técnico Científico - PUC-Rio 
Todos os direitos reservados. É proibida a reprodução total ou parcial do trabalho sem autorização da universidade, do autor e do orientador.

\section{Carlos Cesar Laufer}

Graduou-se em Engenharia Eletrônica pela PUC-Rio, em 1975. Como projetista de software, trabalhou por dez anos na Cobra Computadores Brasileiros S. A., tendo participado da equipe que desenvolveu o sistema operacional para o computador Cobra-500, pioneiro no país. Obteve o título de Mestre em Informática, pela PUC-Rio, em 1994. Trabalhou por três anos em projetos de educação a distância no Laboratório de Engenharia de Software da PUC-Rio.

Ficha Catalográfica

Laufer, Carlos Cesar

Contract Oriented Web Services Model (COWS) - Um Modelo Baseado em Contratos para Suporte a Processos de Negócios na Web / Carlos Cesar Laufer ; orientador: Daniel Schwabe. - 2007.

154 f. : il. ; $30 \mathrm{~cm}$

Tese (Doutorado em Informática) - Pontifícia Universidade Católica do Rio de Janeiro, Departamento de Informática, Rio de Janeiro, 2007.

Inclui referências bibliográficas.

1. Informática - Teses. 2. Web Services Semânticos. 3. Web Services. 4. E-Business. 5. Engenharia de Software. I. Schwabe, Daniel. II. Pontifícia Universidade Católica do Rio de Janeiro. Departamento de Informática. III. Título.

CDD: 004 


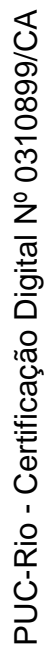

A meus pais. Pelo amor e pela orientação. 


\section{Agradecimentos}

Ao meu professor orientador, Daniel Schwabe, pelo incentivo, pela amizade, pela inteligência, pela crença no meu trabalho e pelo bom humor.

Aos professores membros da banca, pela aceitação do convite de participação na avaliação deste trabalho.

Aos professores José Ricardo Bergmann, Marcus Vinicius Soledade Poggi de Aragão, Clarisse Sieckenius de Souza e Marco Antonio Casanova, por terem me permitido retomar este caminho.

À PUC-Rio, pela minha formação acadêmica e pelos auxílios concedidos.

Ao Digital Enterprise Research Institute (DERI), Galway, pela oportunidade de estabelecer contato com as pesquisas recentes no exterior e pelos auxílios concedidos.

Aos funcionários da secretaria do Departamento de Informática, Ruth, Emanuelle e Alex, pelo suporte administrativo.

À Gabriela, meu amor, pela inteligência, pela diversão e pela revisão do texto deste trabalho.

Às minhas irmãs e aos meus irmãos, Gloria, Ana, Beto e José Mauro, pelo amor que nos une e nos fortifica. 


\section{Resumo}

Laufer, Carlos Cesar. Contract Oriented Web Services Model (COWS) Um Modelo Baseado em Contratos para Suporte a Processos de Negócios na Web. Rio de Janeiro, 2007. 154p. Tese de Doutorado Departamento de Informática, Pontifícia Universidade Católica do Rio de Janeiro.

O estabelecimento de um processo de negócios se faz por meio de relacionamentos entre parceiros que têm um objetivo comum. Esses relacionamentos são definidos em contratos, que podem ser explícitos ou implícitos, podem ser verbais ou escritos, e assim por diante. Quando uma pessoa busca um parceiro de negócios, ela procura um parceiro que possa completar um relacionamento definido em um contrato. Para dar suporte a esses processos na Internet (e na Web) é necessário caracterizar todos os seus aspectos, tais como: agentes, contratos, papéis, relacionamentos, interações entre os parceiros, políticas etc. Este trabalho apresenta o Contract Oriented Web Services Model (COWS) um modelo para um ambiente apropriado para diálogos de E-Business, implementados por meio de Web Services. COWS é baseado em contratos bemdefinidos, acordados entre todas as partes envolvidas e que incorpora vários níveis de políticas. Essas políticas podem estar relacionadas a formas de pagamento, qualidade de serviço (QoS), políticas de privacidade, direitos, retorno de produtos, confiança entre parceiros, entre outros. Os contratos podem se referenciar a outros contratos e têm escopo em foros específicos, que contêm políticas globais. Um protótipo, para um ambiente Web com suporte ao COWS, foi implementado para testar os conceitos que estendem o processo de casamento de parceiros, em um processo de negócios. Todos os modelos do COWS foram especificados como ontologias, utilizando-se a linguagem Flora-2.

\section{Palavras-chave}

Web Services Semânticos, Web Services, E-Business, Processo de Negócios, Contrato, Política, Ontologia. 


\section{Abstract}

Laufer, Carlos Cesar. Contract Oriented Web Services Model (COWS) A Semantic Contract Support for E-Business Processes. Rio de Janeiro, 2007. 154p. DSc. Thesis - Departamento de Informática, Pontifícia Universidade Católica do Rio de Janeiro.

Business processes are established via relationships between partners with a common goal. These relationships are specified in contracts, which could be explicit or implicit, oral or written, and so on. When a person searches for a business partner, she is looking for a partner that can fulfill a relationship specified in a contract. To support such processes in the Internet (and in the Web) it is necessary to characterize all of its aspects, such as agents, contracts, roles, relationships, interactions between partners, policies, etc. This dissertation presents the Contract Oriented Web Services Model (COWS) - a model for an appropriate environment for E-Business dialogues, implemented using Web Services. COWS is based on well-defined contracts agreed upon by all concerned parties and incorporates various levels of applicable policies. These policies can be related to payment methods, quality of service $(\mathrm{QoS})$, privacy policies, rights, products return, trust, etc. Contracts may refer to other contracts and are valid within forae, which have default global policies. A prototype web environment supporting COWS has been implemented to test the concepts that extend the discovery process. All COWS models have been specified as ontologies, using Flora-2.

\section{Keywords}

Semantic Web Services, Web Services, E-Business, Business Process, Contract, Policy, Ontology. 


\section{Sumário}

1 Introdução 16

$\begin{array}{ll}\text { 1.1. Contrato } & 19\end{array}$

1.2. O Problema 21

1.3. Motivação 22

1.4. Objetivos da Pesquisa 23

1.5. Estrutura da Tese 23

2 Serviços 25

2.1. Componentes 27

2.2. Arquitetura Orientada a Serviço 28

2.3. Web Services 30

2.3.1. Web Services Architecture 33

2.4. Web Services Semânticos 35

2.4.1. OWL-S 36

$\begin{array}{ll}\text { 2.4.2. WSMO } & 37\end{array}$

2.4.3. WS-BPEL 38

2.4.4. METEOR-S 39

2.4.5. SWSF 40

3 Negócios $\quad 42$

3.1. Diálogos de E-Business 45

3.1.1. RosettaNet 46

3.2. Contratos 47

3.2.1. FIPA 52

3.2.2. WS-CDL 53

3.2.3. ebXML 54

3.3. Cadeia de Contratos 56

3.4. Políticas

3.4.1. WS-Policy 58

3.4.2. WS-Agreement 59 
3.4.3. Rei 60

$\begin{array}{ll}\text { 3.5. Confiança } & 61\end{array}$

4 Contract Oriented Web Services Model (COWS) 63

4.1. Notation $3-\mathrm{N} 3 \quad 66$

4.2. Modelo Conceitual 68

4.2.1. Contrato 69

4.2.2. Agente 72

4.2.3. Papel 74

4.2.4. Política 76

4.2.5. Confiança $\quad 77$

$\begin{array}{ll}\text { 4.2.6. Foro } & 78\end{array}$

4.3. O Ambiente Operacional 79

4.3.1. Casamento de Parceiros 79

4.3.2. Fase de Execução $\quad 81$

5 Implementação $\quad 84$

5.1. Ontologias 84

5.1.1. Contrato 84

5.1.2. Publicação 85

$\begin{array}{ll}\text { 5.1.3. Busca } & 87\end{array}$

5.1.4. Resultado de Busca 89

5.1.5. Foro 90

5.2. Ambiente de Testes 90

5.3. Política 93

5.3.1. Exemplo 96

5.3.2. Sobreposição de Políticas na Busca 98

5.4. Confiança 98

5.5. Cadeia de Contrato 103

5.5.1. Cadeia de Contrato Automática 105

5.6. Casamento Funcional 106

6 Cenários de Aplicação 114

6.1. Contract Farming 114 
6.1.1. Sobreposição de Política na Busca 117

6.1.2. Foro 117

6.1.3. Múltiplos Contratos e Múltiplos Papéis 119

6.2. Amazon 121

6.2.1. Múltiplos Contratos e Múltiplos Papéis 123

6.2.2. Políticas 126

6.3. Musician's Friend 128

6.3.1. Cadeia de Contratos 130

$\begin{array}{ll}\text { 6.3.2. Confiança } & 131\end{array}$

7 Conclusões 134

7.1. Avaliação 134

7.1.1. Expressividade 135

7.1.2. Casamento de Parceiros 136

$\begin{array}{ll}\text { 7.1.3. Ordem de Filtragem } & 137\end{array}$

$\begin{array}{ll}\text { 7.1.4. Contrato } & 137\end{array}$

7.1.5. Encadeamento de Contratos 138

7.1.6. Políticas 138

7.1.7. Foro 139

7.1.8. Fase de Execução 140

7.2. Contribuições 141

7.3. Trabalhos Relacionados 143

$\begin{array}{ll}\text { 7.4. Trabalhos Futuros } & 146\end{array}$

$\begin{array}{lr}\text { Referências Bibliográficas } & 149\end{array}$ 


\section{Lista de figuras}

$\begin{array}{ll}\text { Figura } 1 \text { - Quebra-cabeça } & 16\end{array}$

Figura 2 - Paradigma procurar-vincular-executar de SOA 30

Figura 3 - PIP3A4 - Solicitação de Ordem de Compra 47

Figura 4 - FIPA Contract Net Protocol 53

Figura 5 - Exemplo de encadeamento de contratos 57

Figura 6 - Exemplo de WS-Policy $\quad 59$

Figura 7 - Exemplo de contrato envolvendo três papéis 64

Figura 8 - Exemplo de processo de negócios definido com PIPs

RosettaNet 66

Figura 9 - Modelo Conceitual do COWS 69

Figura 10 - Contrato 70

Figura 11a - Especificação de contrato em N3 71

Figura 11b - Especificação de contrato em N3 72

Figura 12 - Agente $\quad 72$

Figura 13 - Especificação de agente em N3 73

Figura 14 - Papel 74

Figura 15 - Especificação de papel em N3 75

Figura 16 - Política 76

Figura 17 - Especificação de política em N3 77

Figura 18 - Confiança $\quad 77$

Figura 19 - Especificação de confiança em N3 78

$\begin{array}{ll}\text { Figura } 20 \text { - Foro } & 79\end{array}$

Figura 21 - Especificação de foro em N3 79

Figura 22 - Ambiente operacional COWS 80

Figura 23 - Ontologia de contrato do COWS em Flora-2 85

Figura 24 - Exemplo de instância de tipo de contrato 85

Figura 25 - Ontologia de publicação do COWS em Flora-2 86

Figura 26 - Exemplo de instância de anúncio de agentes 87

Figura 27 - Ontologia de busca do COWS em Flora-2 88

Figura 28 - Exemplo de instância da ontologia de busca 88 
Figura 29 - Ontologia de resultado de busca do COWS em Flora-2 89

Figura 30 - Ontologia de foto do COWS em Flora-2 90

Figura 31 - Ativação da shell do Flora-2 91

Figura 32 - Carga do módulo com o COWS 91

Figura 33 - Regra de definição de soluções de uma busca 92

Figura 34 - Exemplo de tela de resultado de busca 94

Figura 35 - Exemplo de política de foro 94

Figura 36 - Anúncio de publicação do agente FedEx 96

Figura 37 - Política de restrição de entregadores do agente Carlos $\quad 97$

Figura 38 - Exemplo 1 de sobreposição de políticas 98

Figura 39 - Exemplo 2 de sobreposição de políticas 99

Figura 40 - Contrato de compra e venda por atacado entre dois parceiros 100

Figura 41 - Anúncio dos agentes Loja das Fábricas e

Fábrica Brastemp 100

Figura 42 - Solicitação de busca do agente Fábrica Brastemp 100

Figura 43 - Anúncio do agente Carlos com o contrato de $\begin{array}{ll}\text { compra e venda por atacado } & 101\end{array}$

Figura 44 - Anúncios de agentes com restrição de confiança 102

Figura 45 - Contrato de compra e venda no varejo entre dois parceiros 103

Figura 46 - Anúncio do agente Loja das Fábricas 104

Figura 47 - Anúncio do agente Carlos 104

Figura 48 - Solicitação de busca do agente Carlos 105

Figura 49 - Ontologia de contrato do COWS em Flora-2 106

Figura 50 - Exemplo de uma publicação em um registro UDDI 107

Figura 51 - Exemplo de uma busca em um registro UDDI 107

Figura 52 - Exemplo de instância de anúncio de agentes com o atributo unspsc 108

Figura 53 - MatchMaker UNSPSC do COWS 108

Figura 54 - Web Service (WSMO) - propriedades não-funcionais 110

Figura 55 - Web Service (WSMO) - precondições 111

Figura 56 - Web Service (WSMO) - condições posteriores 111 
Figura 57 - Web Service (WSMO) - efeitos

Figura 58 - Busca (WSMO) - propriedades não-funcionais 112

Figura 59 - Busca (WSMO) - condições posteriores 113

Figura 60 - Busca (WSMO) - efeitos 113

Figura 61 - Contrato entre agricultores e comerciantes 115

Figura 62 - Anúncio dos agentes Comerciante 1, Agricultor 1 e Agricultor $2 \quad 116$

Figura 63 - Especificação de busca do agente Comerciante 1

Figura 64 - Especificação de busca do agente Comerciante 1 com sobreposição

Figura 65 - Exemplo de política de foro no cenário de Contract Farming

Figura 66 - Contrato entre agricultores, comerciantes e transportadores

Figura 67 - Anúncio dos agentes Agricultor 3, Comerciante 1 e Transportador 1

Figura 68 - Especificação de busca do agente Comerciante 1 em dois tipos de contrato 121

Figura 69 - Anúncios dos agentes no contrato entre dois parceiros 123

Figura 70 - Anúncios dos agentes no contrato entre três parceiros $\quad 124$

Figura 71 - Especificação de busca dos três agentes 125

Figura 72 - Política de meios de pagamento do agente Amazon $\quad 127$

Figura 73 - Política de devolução de produtos do agente Carlos 129

Figura 74 - Anúncio do agente Musician's Friend 130

Figura 75 - Contrato entre dois parceiros com intermediário 131

Figura 76 - Anúncio dos agentes Musician's Friend e

Web International Trading

Figura 77 - Confiança entre os agentes Musician's Friend e

Web International Trading 


\section{Lista de tabelas}

Tabela 1 - Papéis semânticos $\quad 50$

Tabela 2 - Resultado de busca $\quad 89$

Tabela 3 - Resultado de busca do agente Carlos 97

Tabela 4 - Resultado de busca do agente Fábrica Brastemp 101

Tabela 5 - Resultado de busca do agente Fábrica Brastemp (2) 101

Tabela 6 - Resultado de busca do agente Carlos (2) 105

Tabela 7 - Resultado de busca do agente Comerciante 1

Tabela 8 - Resultado de busca do agente Carlos (3) 125

Tabela 9 - Resultado de busca do agente Amazon 125

Tabela 10 - Resultado de busca do agente FedEx 126 


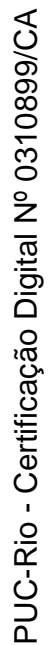

Os Flinstones, os Simpsons, os Jetsons: negócios são sempre negócios. 